\title{
Choice modelling in social networks using stochastic actor-oriented models
}

\author{
Sebastian Pink *, David Kretschmer, Lars Leszczensky \\ Mannheim Centre for European Social Research (MZES), University of Mannheim, Mannheim, Germany
}

\section{A R T I C L E I N F O}

\section{Keywords:}

Stochastic actor-oriented models

Social networks

Siena

Choice modelling

\begin{abstract}
A B S T R A C T
Combining choice modelling with social network analysis, we show how the stochastic actororiented model for the co-evolution of networks and behavior (SAOM) can be used as a powerful statistical framework to empirically analyze network-related choices. We discuss the underlying assumptions of SAOMs and show that they can be interpreted to represent a random utility maximization model (RUM). Network-related choices pertain both to decisions to engage in (or disengage from) specific social relationships and decisions to adapt behavior to that of social contacts. We demonstrate the usefulness of SAOM for the choice modelling community. We further illustrate how SAOM can be used to study network-related choices by providing an exemplary empirical analysis.
\end{abstract}

\section{Introduction}

People make choices concerning a wide array of subjects, such as where to live, which transportation mode to use, or which wine to buy. Conceptually, these choices are based on two principles. First, different alternatives satisfy people's preferences to different extents, thus providing different utility, and people choose the alternative with the highest utility (McFadden, 1973). Second, utility is not tied to the alternatives as such but derives from the combination of their characteristics, attributes, or properties (Lancaster, 1966). If people, for example, think about moving to a different neighborhood, they may consider that this potential new place of residence differs from the current one in terms of commuting time, local facilities, or social composition of its residents, and then choose to move or to stay. Thus, understanding how people weigh these different characteristics is crucial. Stated choice experiments (SCE) constitute a popular means to disentangle the importance of different characteristics (Louviere et al., 2000), and the random utility maximization model (RUM; McFadden, 1973) is a key tool to specify how choice situations can be modelled statistically to estimate the probabilities of choosing each alternative in a given choice set.

However, people do not only make decisions about goods, such as transportation modes or wine, but also about their social relationships. That is, people choose who to befriend, who to collaborate with, or who to gossip about. These relations result in social networks, which can be defined as the web of specific relationships among a group of people, such as friendships in school, co-authors in a scientific field, or gossipers in a firm. From a choice modelling perspective, social networks are interesting for two reasons. First, social networks rarely emerge randomly but, as mentioned above, arise from people's decisions regarding their network partners. In the literature on social networks, these decisions are referred to as the selection of interaction partners (Steglich et al., 2010). The structure of networks, then, is the outcome of individuals' choices of who to connect to. Thus, although the choice to select interaction

\footnotetext{
* Corresponding author. Mannheim Centre for European Social Research (MZES), University of Mannheim, A5, 6, D-68131, Mannheim, Germany. E-mail address: sebastian.pink@uni-mannheim.de (S. Pink).
} 
partners reflects only one specific choice process among many others, it is particularly interesting and challenging due to the inherent interdependency of choices in social networks. The reason is that social networks do not suddenly pop up such that a group of people selects their interaction partners at the same time. Rather, social networks emerge over time through a sequence of people selecting their interaction partners. This renders people's choices inherently dependent on the previous choices of other people because these choices change how attractive social relationships to particular people become. Thus, understanding choices regarding the selection of interaction partners, on which we focus in the following, is inextricably linked to the idea of interdependency of choices and, consequentially, to the evolution of social networks. One outcome of these selection processes is, for example, the size of personal networks, which has been assessed previously based on hybrid choice models using the relationship strength to predict retention and loss of social contacts (Calastri et al., 2018). Second, irrespective of how social networks formed and evolved, they are often important for choice modelling processes because people are embedded in networks which influence their non-network related individual behavior and decisions. For example, people have been shown to be influenced by their social contacts in their political voting choices (Reed, 2015). These effects of network partners on individual outcomes are referred to as influence, and understanding how and why people's choice behavior is influenced by their network partners is another key task for both network researchers and choice modellers.

Studying network-related choices, however, is complicated by the fact that both selection and influence result in the same structural outcome of similar people being connected to each other. For example, do adolescents start drinking alcohol because their friends do so, or do drinkers befriend other drinkers (Osgood et al., 2013)? Answering such questions requires separating selection (i.e., choosing network partners based on their characteristics) from influence (i.e., choosing to change one's own behavior because of network partners). One statistical approach to study choices in a social network context and to separate selection from influence is the so-called stochastic actor-oriented model for the co-evolution of networks and behavior (SAOM, Snijders et al., 2010; Steglich et al., 2010). Though SAOMs are specifically suited to studying choice processes in network-related settings, the literature on choice modelling and social networks have so far been largely decoupled (but see Arentze et al., 2013; Calastri et al., 2018; Reed, 2015). In fact, a core critique of research on decision-making is that it often fails to take social context into account (Bruch and Feinberg, 2017; Maness, 2020), an important part of which is people's embeddedness in social networks. Furthermore, this decoupling is surprising because rational choice arguments might be a common basis of modelling decision-making processes (Hess et al., 2018) as they feature in both literatures. Several authors have pointed to the conceptual alignment of the SAOM modelling strategy with rational choice considerations (Amati et al., 2015; Snijders, 1996; Van de Bunt et al., 1999) and SAOMs have been applied to substantive questions related to rational choice arguments (Leszczensky and Pink, 2015).

In this paper, we aim to increase the mutual appeal between the fields of social network analysis and choice modelling. In order to do so, we focus on the SAOM, a well-established method of longitudinal social network analysis (for the main introduction, see Snijders et al., 2010). We show that a SAOM can be interpreted as a type of choice model, particularly as a random utility maximization model (RUM; McFadden, 1973, 1986), in which network features constitute the characteristics that shape people's utility and people make network-related choices by utility maximization. To prepare this comparison, we first provide a short description of the random utility maximization model. Afterwards, we provide a basic intuitive understanding of how SAOMs work. Thereafter, we engage in a more formal discussion of how SAOMs model choices and show how the model's foundations relate to random utility maximization models. Then, we illustrate the usefulness of SAOMs for choice modelling in a network context by discussing an exemplary application that shows how academic achievement affects friendship formation among adolescents and how adolescents' friendships influence their academic achievement. We close with a short summary of the benefits of SAOMs for choice modelling and an outlook on the further possibilities using SAOMs.

\section{Modelling choices: the random utility maximization model}

A variety of theoretical approaches to decision-making assume that choices are associated with specific consequences for the decision maker, who then chooses the alternative that provides the most utility or satisfaction to her (Kroneberg and Kalter, 2012; Liebe et al., 2018). According to Lancaster's (1966) characteristics theory of value, the utility of choosing specific goods does not derive from these goods per se but depends on the properties or characteristics of each good. However, this utility is not conceived to be deterministic. It acknowledges both that people may err about the utility of alternatives and that non-observed traits of decision-makers or goods may contribute to the utility. These ideas are incorporated by the random utility maximization model (RUM; McFadden, 1973). Formally, an actor $i$ 's total utility $U$ from an alternative $j$ is

$$
U_{i j} \quad \sum_{k 1}^{K} \beta_{k} x_{k i j} \quad \varepsilon_{i j}
$$

a linear combination of attributes $x_{k i j}$, weighted by their importance $\beta_{k}$, and a random utility component $\varepsilon_{i j}$. Assuming that the latter has a type 1 extreme value distribution (also called Gumbel distribution), the contributions $\beta_{k}$ of the attributes to utility can be estimated from empirical data in a multinomial logistic regression framework (McFadden, 1973). Given that people are assumed to maximize their utility, the probability that actor $i$ chooses alternative $j$ from her choice set of $1, \ldots, J$ alternatives is

$$
\operatorname{Pr}_{i j} \frac{\exp \sum_{k{ }_{1}}^{K} \beta_{k} x_{k i j}}{\sum_{h{ }_{1}}^{J} \exp \sum_{k{ }_{1}{ }_{1} \beta_{k} x_{k i h}}} .
$$

This probability is oftentimes interpreted as an individual's stochastic preference for a specific alternative. In empirical 
applications, however, the preference for a specific alternative constitutes a revealed preference as the decision-making process itself remains hidden to the researcher in this kind of model, as only the decision itself is observed (Liebe et al., 2018).

In the following sections, we will return to this RUM setup after an intuitive introduction to SAOMs in order to show the commonalities and differences between the SAOM and the RUM and thereby discuss to which extent SAOMs may be conceived as choice models.

\section{The stochastic actor-oriented model}

The stochastic actor-oriented model for the co-evolution of networks and behavior (SAOM) is a statistical model to investigate the evolution of social networks between two or more discrete time points, which was developed by Tom A. B. Snijders and colleagues (Snijders, 2001; Snijders et al., 2010). ${ }^{1}$ It provides methodological solutions to one of the major challenges of investigating the evolution of social networks, namely that its members select their network partners while simultaneously being influenced by them.

SAOMs have been applied to study manifold social phenomena and networks such as scientific collaboration networks (Ferligoj et al., 2015), interbank credit (Finger and Lux, 2017), cultural tastes (Lewis et al., 2008), arctic expedition networks (Block et al., 2018), international weapons agreements (Kinne, 2016), or adolescents' friendship networks in school (Leszczensky and Pink, 2015). SAOMs model the interdependency of choices in a social network by simulating a sequence of decisions in which individuals (also called "actors") shape the evolution of the network. ${ }^{2}$ In addition, the network itself and the social relationships that people maintain can affect other individuals' choices as well. These network effects do not only concern network-related choices, but can also impact individuals' personal characteristics (such as attitudes and behavior) through influence processes.

SAOMs implement this interplay of how people form social relationships and how these relationships influence them as two parts of the same model (Steglich et al., 2010). This is a great advantage because while previous choice modelling applications highlight the importance of social influence in choice models (e.g., Walker et al., 2011), they do not systematically model the selection of the social contacts that frequently accompanies this influence (e.g., Paez and Scott, 2007), but rather analyze either selection (e.g., Calastri et al., 2018) or influence (e.g., Reed, 2015). In SAOMs, the selection part models changes in the social network that result from people's relationship choices; the influence part models changes in people's individual characteristics, due to their relationships to other people, i.e., how they choose to alter their characteristics in response to those surrounding them. If researchers are only interested in explaining relationship choices (by studying the formation and change of a given social network), the selection part may be sufficient. However, if they are (also) interested in how a social network affects individual characteristics (behaviors and attitudes such as desires, beliefs, identification, effort, or reputation) the model also has to include the influence part. Jointly modelling selection and influence is particularly important if the underlying processes result in the same outcome. The reason is that connected actors may be similar to one another in a characteristic because they chose similar interaction partners or adapted to their interaction partners. As we illustrate below, SAOMs can be used to disentangle these processes.

\subsection{Modelling choices about social relationships (selection)}

The heart of a SAOM is the modelling of individuals' decisions that, in concert, drive the evolution of social networks by forming and dissolving social relationships. SAOMs assume that individuals make these choices in a myopic manner, meaning that they only consider immediate, self-referred consequences of their decisions for their local environment. This so-called myopic stochastic optimization (Snijders, 2004) constitutes a simplification and implies that network members do not decide strategically (Indlekofer, 2014: 36; Steglich et al., 2010). Longer-term objectives, indirect rewards, learning from the past or coordination with others are thus ignored in the modelling process (Brandes et al., 2012: 300; Snijders and Steglich, 2015: 234).

As will be shown when considering relations to the random utility model, SAOMs can be motivated from a rational-choice approach (Snijders, 1996), but are generally flexible regarding behavioral assumptions (Snijders and Steglich, 2015: 225f.), and thus not necessarily married to a specific theory of action or decision-making. Taken together, put very simply, in the SAOM approach, every time individuals have the opportunity, they choose what seems best for them in that very moment. SAOMs therefore do not require strong assumptions about perfectly rational actors that are aware of the complete ramifications of their actions.

An individual's choice can have three potential outcomes: the creation of a social relationship, the dissolution of a social relationship, or the maintenance of the status quo. The decision for one of these alternatives happens in a so-called "mini-step". Strictly speaking, however, the choice itself is not a choice between individual options but between potential states of the social network that a member of the network may induce through his or her action. Put differently, the choice set contains all different potential states of the social network that an actor may create by a single decision. This set then contains all states that might be realized through adding a tie to any actor to whom the focal actor is not yet connected to, all states that originate from dissolving an existing tie, and the status quo. Each of these potential states in the choice set is evaluated using a function that is based upon the characteristics of the potential states of the network.

SAOMs assume that, when making their choices, individuals are fully informed about the current state of the social network, i.e.,

\footnotetext{
1 SAOMs are implemented in the simulation-based estimation method "SIENA" ("Simulation Investigation for Empirical Network Analysis").

2 For the sake of brevity, we refer to decision-making units as "people" or "individuals". Note, however, that SAOM can also be applied to groups of people who act in concert and who are related to other groups of their kind, such as organizations or firms. For readers more interested in the preconditions of a social network analysis, we refer to a short outline in Appendix A.
} 
the social relationships among all actors as well as all actors' relevant characteristics (Snijders et al., 2010; Veenstra et al., 2013). Only given this assumption, people may be modelled as if they envisioned the set of network states they may generate and deliberated about which to choose. This assumption of full information has consequences for the size of social networks that SAOMs can plausibly be applied to. As the number of people increases, it becomes more likely that people do not know all other people or their relationships among each other (e.g., Dunbar, 1992). While this assumption can be defended in rather small networks, such as a classroom network, it may be violated in larger networks that consist of hundreds of actors, such as a large school.

\subsubsection{Formally modelling choices about relationships: the evaluation function}

The choice between possible states of the social network (i.e., the distinct elements of the choice set) is a discrete choice between nominal alternatives (Snijders, 2001; Snijders et al., 2010). Actor $i$ evaluates a particular state $j$ of the social network with the so-called evaluation function

$$
f_{i j} \beta, s \quad \sum_{k}^{K} \beta_{k} s_{k i j} \quad \varepsilon_{i j} .
$$

Each possible state of the social network that an actor may realize in a mini-step thus has a particular evaluation function value $f_{i j} \beta$, $s$, which consists of a deterministic and a stochastic component. The deterministic component is a weighted sum of a series of network characteristics in the form of network statistics $s_{k i j}$ and the importance of these statistics, as reflected in the direction and strength of the associated coefficients $\beta_{k}$. The network statistics $s_{k i j}$ are counts of network configurations that are considered to be relevant for people's evaluation of a network state. If they, for example, value same-sex relations, they will evaluate network states that contain more samesex relations more positively, implying a positive $\beta$ on the count of same-sex relations $s_{i j}$. This deterministic component is complemented by a stochastic error term $\varepsilon_{i j}$ that captures idiosyncratic errors and influences on the evaluation of network states that are not modelled explicitly. This error is assumed to be Gumbel-distributed (Snijders, 2001). Actors then choose the network state $j$ with the highest evaluation function value, which they implement by creating a new tie, dissolving an existing tie, or maintaining the status quo. Fig. 1 illustrates this process of making a choice regarding outgoing ties.

The SAOM mini-step choice process closely resembles the classic random utility maximization setup, as can be seen by comparing equations (1) and (3). Interpreting the statistics $s_{k i j}$ in the deterministic component of the SAOM evaluation function as network attributes that determine actors' utility from a given network state, the evaluation function is conceptually identical to the classical random utility maximization model $U_{i j} \sum_{k 1}^{K} \beta_{k} x_{k i j} \quad \varepsilon_{i j}$ with $x_{k i j} \quad s_{k i j}$. In both the classic RUM and the SAOM, the deterministic component of utility is complemented by a Gumbel-distributed stochastic error term, such that both models imply a multinomial logit model for estimation on empirical data (Steglich et al., 2010: 49). Like the different alternatives in the RUM model (see equation (2)), an actor $i$ chooses a specific network state $j$ with a probability of

$$
\operatorname{Pr}_{i j} \frac{\exp \sum_{k{ }_{1} \beta_{k} s_{k i j}}^{J}}{\sum_{h{ }_{1}}^{J} \exp \sum_{k{ }_{1}{ }_{1} \beta_{k} s_{k i h}}} .
$$

This conceptualization of SAOMs in terms of random utility models demonstrates that SAOMs can be understood as choice models, which model social relationships as the outcome of preference-based decision-making processes (also see Calastri et al., 2018; Van de Bunt et al., 1999). However, as SAOMs are also generative statistical models designed to study the evolution of social networks, the evaluation function will frequently contain network attributes that fit less well with a preference interpretation for those attributes but that help to more appropriately model the structure of the networks. As outlined later, such network attributes refer to structural properties of the network (e.g., an outdegree effect modelling the general tendency to have a number of social relationships) or key sources of opportunities for interaction.

Note that the potential network states actors can choose between in any given mini-step do not necessarily differ in all of the specified network attributes $s_{k i j}$. The importance $\beta$ of the network attributes is always estimated taking into account opportunities to affect them with a given choice (e.g., Block and Grund, 2014). In other words, although actors may have preferences for certain relationship configurations, they may not always be able to realize them due to a lack of opportunities. Estimates of $\beta$, however, are not biased by the opportunity structure individuals face in their network choices.

\subsubsection{The evaluation function and the attributes of network states}

What types of attributes or characteristics of social networks may SAOMs incorporate? As indicated earlier, the values of the evaluation function derive from network statistics $s_{k i j}$ that represent different types of attributes (or effects). ${ }^{3}$ These attributes represent researchers' theoretical and empirical expectations about the determinants of individuals' network-related choices. Three types of attributes may be included. First, attributes may represent individual covariate effects, which refer to effects of network members' individual characteristics, such as their gender or socio-economic status. These attributes take the form of ego or alter affects and model whether the frequency of sending or receiving network ties varies according to the characteristic considered, i.e., whether actors

\footnotetext{
${ }^{3}$ More detailed descriptions and their mathematical formulations, also for the effects of the influence part discussed later, may be found in Ripley et al. (2019), Snijders et al. (2010), or Snijders (1996).
} 


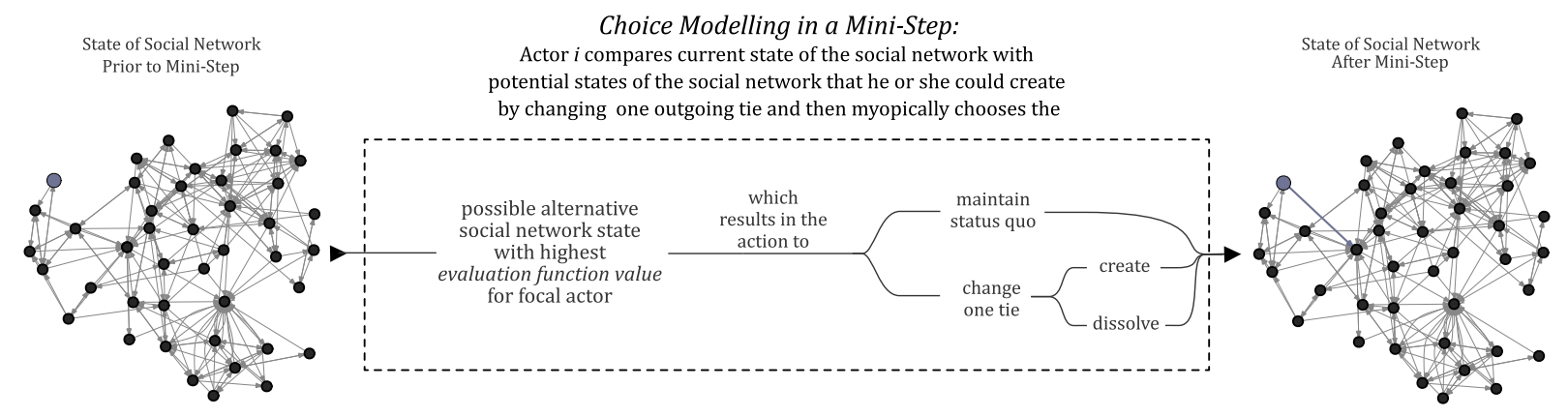

Fig. 1. Illustration of modelling network choices (selection) using the stochastic actor-oriented model.

with specific attributes are more active or popular than others.

Second, attributes that are based on the dyadic combinations of ego's and alter's characteristics are called dyadic covariate effects. A same dyadic covariate effect allows to test whether actors who share a characteristic are more likely to have ties to each other than actors who differ in that characteristic. Such similarities between individuals are particularly important because of the well-established tendency to associate with similar others (McPherson et al., 2001). This tendency does not only extend to demographic categories such as gender or ethnicity, but also to various kinds of behavior, attitudes, or opinions. For instance, social relationships often are more likely to be created between people who hold similar opinions or who engage in similar behaviors.

Third, effects of the state of the social network on the formation of ties may be taken explicitly into account using structural effects. These pertain to configurations of ties that may affect whether or not people form ties. Such structural configurations may, for example, be on the dyadic level (i.e., between two people) or on the triadic level (i.e., between three people) with configurations such as reciprocated dyads (i.e., reciprocal directed ties between two people) or closed triads (e.g., transitivity, a person becoming friends with a friend's friend). Both the tendency to reciprocate incoming ties and to close triadic structures are behavioral tendencies that are well-documented across a wide range of social networks (Ripley et al., 2019).

Returning to the specification of effects in the evaluation function (equation (3)), all of these attributes are incorporated into the SAOMs as counts $s_{k i j}$ of the respective network attributes $k$ in a given network state $j$. For a reciprocity effect, for example, the respective count is the number of reciprocal ties in network state $j$. This count varies between different potential network states because, by forming an additional tie, an actor is able to reciprocate one (more) friendship nomination in some networks but not in all. Together with an attribute's parameter value $\beta_{k}$, the count of the network configurations then determines a network state's value on the evaluation function.

In the literature applying SAOMs, the previously outlined decision-making heuristic concerning relationship formation has been described in different ways. For example, it has been interpreted as the attractiveness of particular network states compared to others (Veenstra et al., 2013), as representing the network members' "short-term objectives" (Snijders et al., 2010: 47), as the "behavioral 'rules"' that people will "probabilistically follow" (Lewis et al., 2008: 1697), or as possible network states' "potential to increase or decrease [the] level of satisfaction" with the current state (Finger and Lux, 2017: 241).

\subsection{Modelling changes in personal characteristics (influence)}

So far, our considerations have focused on choices concerning social relationships to network partners. However, as outlined earlier, a unique feature of SAOMs is that they also allow to investigate how social networks influence people's behavior, choices, and personal characteristics in non-network related domains (Steglich et al., 2010). In an influence part that is modelled jointly with the selection part that we have discussed above, SAOMs allow to model changes in actors' behavior and other characteristics, subsumed under the term of "behavior" variables.

In particular, the influence part allows to capture how characteristics of the social network shape actors' non-network related behavior. As outlined earlier, this may be a valuable extension of choice modellers' toolbox as SAOMs can help address critiques of research on decision-making being inattentive to actors' social context (Bruch and Feinberg, 2017), an important part of which is represented by actors' social networks. For example, SAOMs could be used to study how actors' choices of transportation modes or consumer products depends on the characteristics of their social networks and the attributes of their network partners. Such effects of social networks are of particular interest in situations in which the behavior of actors tends to be influenced by the corresponding behavior of their network partners, i.e., when there are social influence effects.

A conceptual advantage of SAOMs is the possibility to simultaneously model network and behavioral evolution. This allows to separate selection from influence effects. This is particularly important in situations with network autocorrelation (Doreian, 1989)_an overlap of networks and personal characteristics - that can originate from both selection and influence (Steglich et al., 2010). In case of network autocorrelation, it is essential to account for selection effects to assess how social networks shape individual behavior through influence processes. Similarly, if the main interest is in how individuals choose their network partners, it is essential to identify these selection effects net of influence. SAOMs are unique in providing the possibility to separate both types of effects because they model both network evolution and behavior change jointly, with both model components being estimated simultaneously. The selection part 
assesses whether similarity in characteristics drives tie formation; the influence part investigates whether actors who have relationships with one another assimilate in their characteristics.

Statistically, changes in the behavioral characteristic(s) are modelled similarly to changes in the structure of the social network. This additional modelling step usually requires an ordinal measure of the behavioral characteristic in question, though extensions to continuous measures have been proposed recently (Niezink et al., 2019). In mini-steps that are conceptually similar to those introduced for relationship formation above, actors' choices with regard to the behavioral characteristic consist of three alternatives: an increase in the behavioral characteristic (by one unit), a decrease, or no change of the value. In other words, people may make either upward or downward moves on the variable's scale or stay where they are. Again in line with the network part of the model, the choice to change the value on the scale is modelled with an evaluation function that captures systematic effects of individual and network characteristics on choice behavior as well as a random term. In a mini-step, members of the network, then, depending on their current value, are more likely to choose a neighboring value on the scale if the evaluation function value of that behavior variable's value is higher (Snijders et al., 2010; Steglich et al., 2010). Again, the setup is conceptually identical to a random utility approach and is empirically estimated using a multinomial logit model.

In terms of model specification, two default parameters are always included in the behavior component of a SAOM to model the general tendency of the behavioral distribution: The linear shape effect shows whether members of the social network generally strive for higher values on the scale of the behavioral variable. The quadratic shape effect indicates whether this is a self-reinforcing tendency (Veenstra et al., 2013). Effects of individual covariates (such as gender, ethnicity, etc.) can be included to model that behavioral choices may differ according to individual characteristics. Importantly, this part of the model can also test whether people are influenced by their social contacts, using influence effects of the personal network that model assimilation in the behavior of connected individuals. One popular specification is the average similarity effect, which measures whether actors tend to adjust their behavioral value to the average of those who they are connected to (Ripley et al., 2019). This possibility to test for whether the contacts people have influence their behavior is one of the great strengths of SAOMs.

\subsection{Parameter estimation and the simulation of the evolution of the social network and behavior}

As shown in the preceding section, the heart of any SAOM is the evaluation function, a linear combination of effects and the coefficients associated with these effects. But how to arrive at estimates for the coefficient $\beta_{k}$ of an effect $s_{k i j}$ ? Being generative models of network evolution, SAOMs are longitudinal models that need information on social network structure for at least two points in time (Snijders et al., 2010). In the standard Methods of Moments estimation approach, SAOMs take the initial empirical network state as an input and, from there, use a simulation approach to model the process that structurally transforms the network observed initially into the empirical network observed at the next point in time. The actual process of network evolution is not observed empirically due to the panel data structure and thus remains unknown to the researcher. SAOMs simulate this evolution process by repeatedly giving network members opportunities for tie formation in the so-called mini-steps, as discussed previously. The frequency of individual actors making a choice is determined by a rate parameter that is estimated from the empirical data. The changes in these mini-steps are the key processes of the SAOM estimation, as they alter the social network step by step, thus constituting the sequence of interdependent choices driving network change. ${ }^{4}$ In this sequence of decisions, actors' choices feed back into other actors' choice sets. By altering the state of the network, albeit in only one tie, an actor changes the set of possible network states a subsequent actor can generate through his or her own choice of network partners as well as the evaluation of these states. Therefore, each subsequent actor's choice of a network state is contingent on the choices of the previous actors. Fig. 2 depicts this process in a stylized way.

Statistically, the trajectory of simulated choices (i.e., mini-steps) is handled as a continuous-time Markov chain (Snijders et al., 2010). This means that each observed state of the network is conditional on the previously observed state of the network, with no additional effects of preceding states. Given the interdependence of choices, the order of the mini-steps is consequential for the final simulated network, such that the simulation process is not conducted once but repeated multiple times. The goal of the simulation procedure is to arrive at simulated networks that resemble the empirically observed network as closely as possible in terms of the network statistics that are spelled out in the model specification. For example, if researchers assume that a preference for others that have the same sex (i.e., gender homophily), reciprocity, and transitive closure are driving forces of the evolution of a social network, they can add related effects as covariates in the model specification by including the number of same-sex ties, the number of reciprocal ties, and the number of transitive triads as network statistics in the evaluation function.

The SAOM estimation process then starts with repeatedly allowing randomly selected actors to make choices regarding network partners according to the mini-steps introduced above. After the simulation run, the (average) number of same-sex ties, the number of reciprocal ties, and the number of transitive triads produced by the simulation model with parameters $\beta_{k}$ are compared to the counts observed in the actual empirical network at that point in time. If these counts are too far from those obtained in the simulated networks, the simulation is repeated after parameter values $\beta_{k}$ have been updated to reflect the incorrect representation of the network characteristics in the simulation. For example, if the initial guess for the parameter for same-sex relationships is close to zero, while the actual network is characterized by many same-sex relationships, the simulated networks will contain few same-gender ties relative to

\footnotetext{
${ }^{4}$ In the following paragraphs, our considerations focus on explaining estimation for the selection part of the model, which captures network evolution. As outlined earlier, estimation for the influence part is analogous, with similar mini-steps modelling change in the behavioral variable(s). In models that contain both a selection and an influence part, the estimation of both parts happens simultaneously, with actors being either chosen for a mini-step that involves either behavioral change or network change. For more details, we refer readers to Ripley et al. (2019).
} 


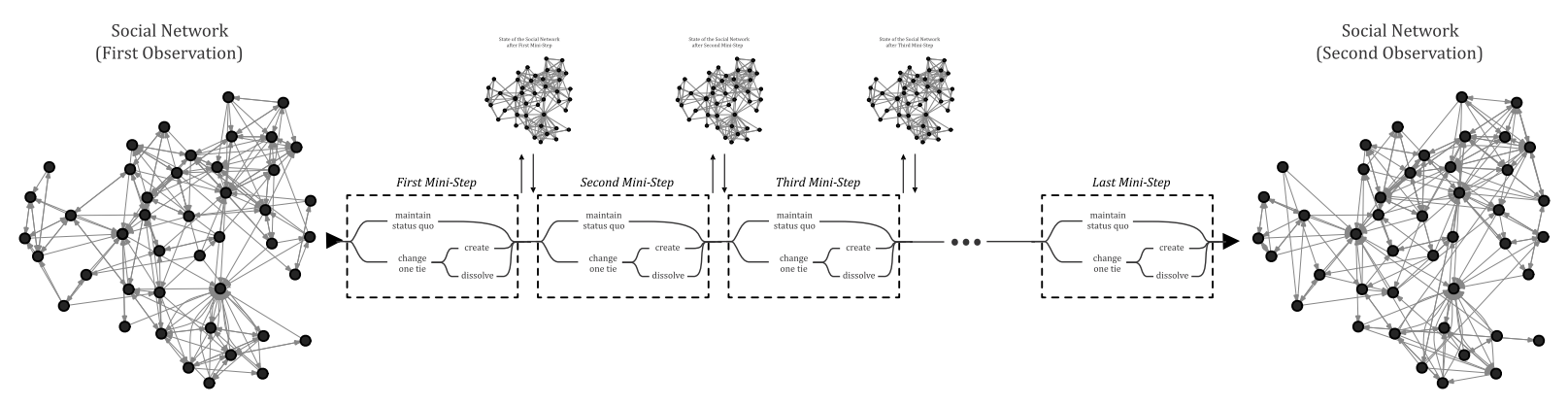

Fig. 2. Illustration of modelling interdependent individual choices using SAOMs.

the actual empirical network. In that case, the coefficient associated with same-gender ties will be increased in subsequent simulations. This updating process is repeated until the simulated networks sufficiently resemble the empirical network in all of the characteristics considered in the model specification. Once this condition is fulfilled, the model is said to have converged, with a specific set of coefficients found through iterative simulation. ${ }^{5}$ The resulting coefficients, as in other nonlinear choice models, can be interpreted in terms of their sign and significance, but not in terms of the size of the substantive effect on probabilities for relationship formation (Mood, 2010). However, both assessing the relative importance of effects (Indlekofer, 2014) or building contrasts of interest (Leszczensky and Pink, 2019) may provide means to more closely interpret the weight of the effects researchers are particularly interested in. ${ }^{6}$

Contrary to statistical models conventionally applied in the choice modelling literature, formal proofs for desirable statistical properties are unavailable for SAOMs due to their mathematical complexity. For example, there are no formal results on the identifiability (Rothenberg, 1971) of SAOMs. However, there is strong suggestive evidence for SAOMs being identified models (Ripley et al., 2019: 16). Internally, SAOMs are built on generalized linear models for actor choices (and the timing of choices). Given this foundation in generalized linear models, SAOMs can be expected to be identified unless redundant effects are included (Niezink et al., 2019; Niezink and Snijders, 2017).

\section{Exemplary application: academic achievement and friendships in school}

To illustrate how to implement, estimate, summarize, and interpret the results of SAOMs, we provide an exemplary application of friendship making and academic achievement in the school context. The following analysis does not constitute an independent empirical contribution; it solely serves illustrative purposes aiming to better understand SAOMs. As such, it is a simplified version of our analysis provided in Kretschmer et al. (2018), which investigated a prominent question in the educational sciences: Does observed clustering according to academic achievement arise because adolescents select friends with similar academic achievement, or do friends influence their friends' academic achievement (e.g., Kandel, 1978)? In what follows, we use this example to demonstrate the usefulness of SAOMs for modelling the preference for certain social relationships, i.e., peers with certain academic achievement as friends, and for assessing whether social networks shape individual choices regarding behavior, i.e., academic achievement.

\subsection{Data}

We use data from the first three waves of the Friendship and Identity in School (FIS) project (Leszczensky et al., 2015). FIS is a longitudinal study on student friendship networks conducted in nine secondary schools in the German federal state of North Rhine-Westphalia. In each school, all students belonging to the fifth, sixth, and seventh grade (i.e., academic year) were surveyed. Fifth-graders were aged 10-11, sixth-graders 11-12, and seventh-graders 12-13 years. The first wave of data collection took place in April 2013, with subsequent waves conducted in January 2014, and October 2014. Overall, 1,668 students from 26 grades were surveyed in the first wave. In the analysis provided here, we focused on grade-level networks for which the student participation rate exceeded 75 per cent in all of the three waves of data collection. Furthermore, we excluded one network with very imprecise parameter estimates. This leaves eleven grade-level networks for the analysis.

Friendship networks were obtained by asking students to nominate up to ten best friends in their grade. Students who did not fill out the questionnaire could nevertheless be nominated so that their ingoing friendships were still recorded. The social networks resulting from this measure can be conveniently represented by adjacency matrices for each of the eleven grades as well as for each

\footnotetext{
${ }^{5}$ The overall maximum convergence ratio indicates the convergence of the estimation algorithm. As a rule of thumb, values smaller than 0.25 are acceptable (Ripley et al., 2019).

${ }^{6}$ We focused on the choice modelling aspect of SAOMs rather than the simulation of network evolution, which is why we refrain from a discussion of the assessment of goodness of fit. However, importantly, besides convergence in parameter estimates, SAOM applications should be accompanied by an inspection of their goodness of fit. Researchers should assess the extent to which the simulated networks that follow from the model specification also adequately represent characteristics of the empirical network that have not been explicitly included in the model specification (for further information, see Ripley et al., 2019).
} 
wave. An adjacency matrix is a square matrix in which the rows and columns denote the students in the grade. The cells provide information about directed friendship nominations. For example, as depicted in Table 1, assume a grade featured 80 students and cell $[2,3]$ entails a one, then student 2 nominated student 3 as a friend. If cell [3,2] entails a one, too, then this friendship is reciprocal. In case there is no friendship nomination between students, the cell features a zero. In case of a student not having participated in the survey (i.e., unit non-response) the entire row of this student features a missing code. However, as ingoing friendships were recorded, too, the column values of this student do not necessarily have to be missing.

We measure academic achievement by students' self-reported grades in the subjects German, English, and mathematics. In the German educational system, grades range from 1 (very good) to 6 (failed). As the worst grade was reported only eight times across all waves and friendship networks, we collapsed it with the second worst grade rather than treating it as a separate category (Fortuin et al., 2016). As a final measure of academic achievement, we use the reverse-coded average grade across the three subjects, rounded to the nearest integer. The scale ranges from 1 to 5, with higher values indicating higher achievement. In SAOM terms, this is the behavior variable.

\subsection{Model specification}

In our exemplary model specification, we use individual covariate effects, dyadic covariate effects, and structural effects in the selection part of the model. We focus on two individual characteristics that may be important for the evolution of adolescents' friendship networks: gender and academic achievement. For gender, we include both ego and alter effects to acknowledge the possibility that activity in the friendship network (the number of ties sent) and popularity (the number of ties received) may vary between boys and girls. Furthermore, we include a same-gender dyadic covariate to assess whether students are more likely to form relationships with same-gender rather than cross-gender peers. For academic achievement, we also include ego and alter effects to assess whether activity and popularity differs according to achievement levels; e.g., students with low (or high) grades might be more (or less) desirable as friends. Most importantly, we also include a similarity effect for academic achievement to test whether students are more likely to befriend schoolmates with similar academic achievement. Finally, we include a dyadic covariate that accounts for whether students share the same classroom, the same neighborhood, or the same elementary schools: all these shared characteristics may provide more opportunities to form friendship relations and are therefore likely to shape friendship formation.

For structural effects, we introduced reciprocity and transitivity via the geometrically weighted edgewise shared partners (GWESP) effect. The reciprocity effect shows whether students reciprocate friendship nominations and the GWESP effect shows whether friends of friends become friends. Furthermore, by default, an outdegree effect is included, which indicates the extent to which friendship nominations are made relative to the size of the network, given that all other effects in the model are zero. This corresponds to a constant in regular regression models and is frequently negative in school-based friendship networks because students have limited capacities to befriend other peers. We also account for a number of additional structural effects (indegree popularity, indegree activity, outdegree activity, and the interaction of reciprocity and GWESP) to more appropriately capture general processes of network evolution (see Ripley et al., 2019), but do not discuss them in detail here.

We also investigate whether students adapt their own academic achievement to their friends' achievement, i.e., whether there are influence processes. We thus conceptualize students' academic achievement as a choice process, with students putting different levels of effort and time into their academic work and thus producing different achievement levels. Given our interest in both whether students choose peers with similar achievement as their friends (selection effect) and whether students assimilate their achievement to their friends' achievement, we can also use our exemplary model to demonstrate SAOMs' ability to separate selection from influence effects. In order to do so, we complement the model's selection part by an influence part that models the determinants of students' academic achievement. Next to the linear and quadratic shape parameters that model the general distribution of academic achievement, we consider variation in achievement according to students' gender. Furthermore, and most importantly, we assess influence effects that originate from friends' academic achievement. These influence effects are accounted for with the achievement average similarity effect, which models whether students assimilate their friends' average achievement levels, i.e., whether they adjust their own achievement towards that of their friends.

We applied this model specification with 20 effects to all of our eleven grade-level networks. As a result, we estimated not one but eleven SAOMs with identical model specifications for different networks from different schools. The estimation was carried out using RSiena 1.2-14 (Ripley et al., 2019). The highest overall maximum convergence criterion value across all eleven SAOMs was 0.15, indicating good convergence. We combined results for the eleven networks using a fixed-effects multivariate meta-analysis (An, 2015; Gasparrini et al., 2012). In this exemplary analysis, we refrained from discussing goodness of fit. We refer interested readers to Kretschmer et al. (2018).

\section{Results}

Before we turn to the SAOM analysis, we provide descriptive information on the 11 grade-level networks we analyze in Table 2 . To show that friends indeed tend to have similar academic achievement, we report Moran's I, a measure of network autocorrelation, for all of our networks. Moran's I assesses whether actors that are directly connected to one another are more similar on some characteristic than randomly selected actors. In all but one of our networks, Moran's I is larger than zero suggesting that friends indeed tend to have similar academic achievement. This similarity may originate from both selecting similar-achieving friends and assimilating friends' achievement.

To run a SAOM analysis that investigates the sources of similarity, we also check whether relationships and achievement change 
Table 1

Adjacency matrix of friendship nominations.

\begin{tabular}{|c|c|c|c|c|c|}
\hline & Student 1 & Student 2 & Student 3 & $\ldots$ & Student 80 \\
\hline Student 1 & 0 & 1 & 1 & $\ldots$ & 0 \\
\hline Student 2 & 1 & 0 & 1 & $\ldots$ & 1 \\
\hline Student 3 & 0 & 1 & 0 & $\ldots$ & 1 \\
\hline$\ldots$ & $\ldots$ & $\ldots$ & $\ldots$ & $\ldots$ & $\ldots$ \\
\hline Student 80 & 0 & 0 & 1 & $\ldots$ & 0 \\
\hline
\end{tabular}

Table 2

Descriptive information on networks.

\begin{tabular}{|c|c|c|c|c|c|c|}
\hline \multirow[t]{2}{*}{ Network \# } & \multirow[t]{2}{*}{ Network Size } & \multirow[t]{2}{*}{ Moran's I Wave 1} & \multicolumn{2}{|c|}{ Jaccard Index } & \multicolumn{2}{|c|}{ Changes in Academic Achievement } \\
\hline & & & Wave $1-2$ & Wave $2-3$ & Wave $1-2$ & Wave $2-3$ \\
\hline 1 & 85 & 0.13 & 0.47 & 0.44 & 12 & 25 \\
\hline 2 & 74 & 0.15 & 0.44 & 0.46 & 11 & 18 \\
\hline 3 & 100 & 0.07 & 0.42 & 0.47 & 26 & 29 \\
\hline 4 & 112 & 0.14 & 0.42 & 0.45 & 18 & 30 \\
\hline 5 & 93 & 0.04 & 0.36 & 0.46 & 13 & 21 \\
\hline 6 & 120 & 0.00 & 0.38 & 0.41 & 38 & 34 \\
\hline 7 & 138 & 0.18 & 0.37 & 0.34 & 48 & 42 \\
\hline 8 & 126 & 0.13 & 0.45 & 0.47 & 44 & 39 \\
\hline 9 & 121 & 0.14 & 0.43 & 0.43 & 37 & 29 \\
\hline 10 & 119 & 0.06 & 0.41 & 0.44 & 34 & 33 \\
\hline 11 & 113 & 0.08 & 0.41 & 0.44 & 33 & 33 \\
\hline
\end{tabular}

over time. Table 2 shows that friendships do change: The Jaccard index, which captures the share of stable friendship relations across subsequent periods is always substantially smaller than 1 , but also not too close to 0 , indicating that some relationships do persist over time. Similarly, Table 2 shows that students' academic achievement changes over time in all of our networks, showing a considerable number of upward and downward moves in the behavior variable.

Given that these preconditions for the SAOM analysis are met, Table 3 shows the main results of our exemplary analysis. We first discuss the results of the selection part, starting with structural effects and then continue with the individual and dyadic covariate effects.

In the selection part of the model, the rate parameters are estimated at around 15, indicating that every actor, on average, has around 15 opportunities to change a friendship tie. Considering structural effects first, note that the coefficient of the outdegree effect is negative and significant, indicating that, in the absence of all other effects, a random pair of students is more likely to not be friends than to be friends. This reflects the fact that students have few friendships relative to the network size, i.e., the network has a low density. The GWESP effect is positive and significant, pointing towards transitive closure: students tend to become friends with the friends of their friends. The reciprocity effect, is significantly positive and indicates that students tend to choose peers as friends who nominated them as friends, too. However, the tendency to reciprocate is weaker in configurations that are transitively closed rather than open (negative interaction effect of reciprocity and GWESP). These findings are in line with previous network studies, thus capturing fundamental network dynamics of friendship networks (e.g., Ripley et al., 2019).

Turning to covariate effects, we first discuss gender differences in friendship formation behavior. Table 1 shows a negative ego effect for gender, but no substantial alter effect. As boys are the reference category in the analysis, these effects indicate that girls send fewer friendship nominations than boys do, and that boys and girls receive the same amount of friendship nominations, i.e., are equally popular. The positive coefficient of the same-gender effect indicates gender segregation in friendship networks, with girls being more likely to befriend other girls rather than boys, and vice-versa. Finally, we find positive and significant coefficients for the same classroom and the same primary school effects, indicating that students are more likely to befriend peers from their own classroom and primary school. Both effects reflect different opportunities to befriend peers.

For academic achievement, ego and alter effects are very close to zero, which indicates that both popularity and activity do not differ by academic achievement. However, we find a positive and significant coefficient for students' similarity in academic achievement. This indicates that students tend to choose those schoolmates as their friends who have an academic achievement that is similar to their own achievement.

To further illustrate the dependence of friendship formation processes on academic achievement, Fig. 3 shows the tendency of students to become friends with schoolmates with different levels of academic achievement. The five horizontal panels differentiate 
Table 3

Meta-analysis of SAOMs.

\begin{tabular}{|c|c|c|}
\hline & \multicolumn{2}{|l|}{ Model 1} \\
\hline & Coef. & SE \\
\hline \multicolumn{3}{|l|}{ Selection Part } \\
\hline \multicolumn{3}{|l|}{ 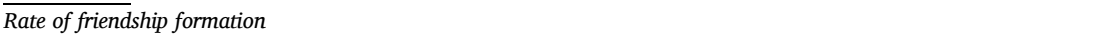 } \\
\hline Period 1 & $16.40^{* * *}$ & $(0.41)$ \\
\hline Period 2 & $14.64^{* * * *}$ & $(0.39)$ \\
\hline \multicolumn{3}{|l|}{ Structural effects } \\
\hline Outdegree & $2.28^{* * * *}$ & $(0.11)$ \\
\hline Reciprocity & $2.31^{* * *}$ & $(0.08)$ \\
\hline Transitivity (GWESP) & $1.56^{* * * *}$ & $(0.03)$ \\
\hline Reciprocity x GWESP & $0.89^{* * * *}$ & $(0.06)$ \\
\hline Indegree-popularity (square root) & $0.17^{* * *}$ & $(0.02)$ \\
\hline Indegree-activity (square root) & $0.47^{* * * *}$ & $(0.04)$ \\
\hline Outdegree-activity (square root) & $0.19^{* * *}$ & $(0.03)$ \\
\hline \multicolumn{3}{|l|}{ Individual-level covariates } \\
\hline \multicolumn{3}{|l|}{ Gender (Ref.: Boy) } \\
\hline Ego & $0.06^{*}$ & $(0.03)$ \\
\hline Alter & 0.01 & $(0.02)$ \\
\hline \multicolumn{3}{|l|}{ Academic achievement } \\
\hline Ego & 0.01 & $(0.02)$ \\
\hline Alter & 0.00 & $(0.02)$ \\
\hline \multicolumn{3}{|l|}{ Dyad-level covariates } \\
\hline Same gender & $0.32^{* * * *}$ & $(0.02)$ \\
\hline Similarity in academic achievement & $0.34 * *$ & $(0.11)$ \\
\hline Same classroom & $0.36^{* * * *}$ & $(0.02)$ \\
\hline Same primary school & $0.07^{* *}$ & $(0.02)$ \\
\hline Same neighborhood & 0.01 & $(0.02)$ \\
\hline \multicolumn{3}{|l|}{ Influence Part } \\
\hline \multicolumn{3}{|l|}{ Rate of behavior change } \\
\hline Period 1 & $0.95^{* * * *}$ & $(0.08)$ \\
\hline Period 2 & $0.99 * * *$ & $(0.08)$ \\
\hline \multicolumn{3}{|l|}{ Trend } \\
\hline Linear shape & 0.09 & $(0.06)$ \\
\hline Quadratic shape & 0.02 & $(0.07)$ \\
\hline \multicolumn{3}{|l|}{ Individual-level covariates } \\
\hline Gender (Ref.: Boy) & 0.03 & $(0.08)$ \\
\hline \multicolumn{3}{|l|}{ Personal network attributes } \\
\hline Average similarity & $3.56^{* * * *}$ & $(0.85)$ \\
\hline
\end{tabular}

Note: Reported coefficients stem from fixed-effects multivariate meta-analysis based on eleven individual network SAOMs; all SAOMs .converged; ${ }^{\dagger} p<0.10,{ }^{*} p<0.05,{ }^{* * *} p<0.01,{ }^{* * * *} p<0.001$.
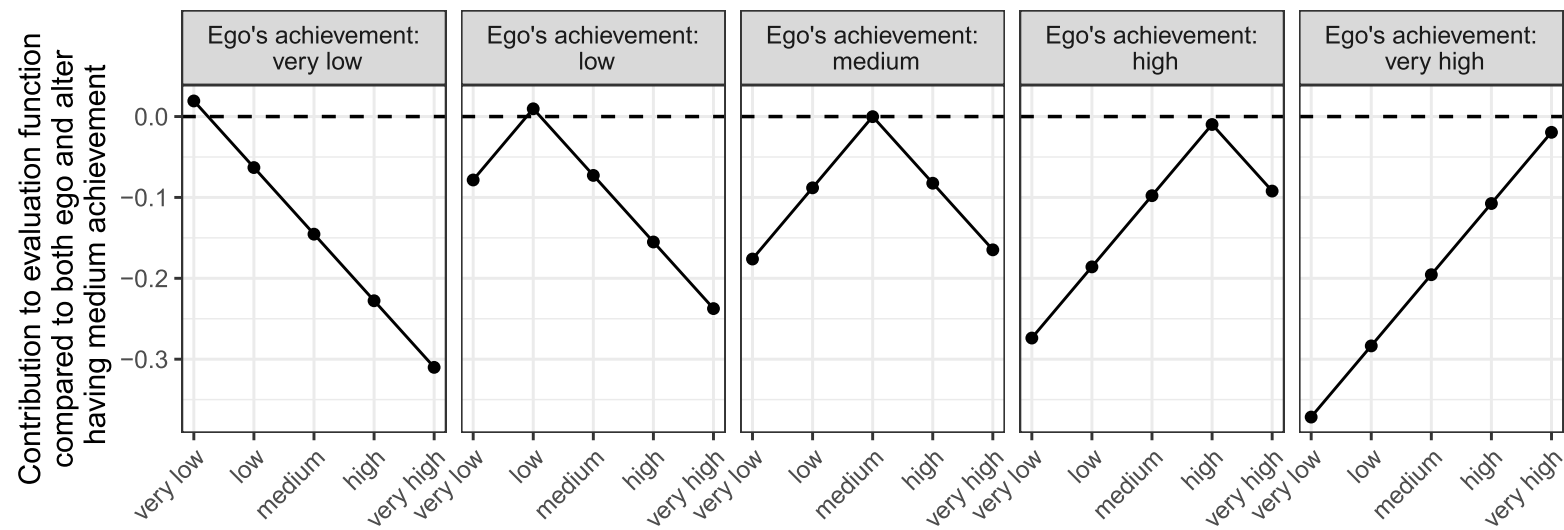

Alter's achievement

Fig. 3. Academic achievement and friendship formation.

focal students ("egos") with different levels of academic achievement. The horizontal axis considers different levels of academic achievement of peers the focal individuals can potentially become friends with ("alteri"). Finally, the vertical axis shows contributions to the evaluation function of the specific combinations of the effects related to academic achievement (ego, alter, and similarity in 
academic achievement; see Table 3). The reference group (i.e., the dashed zero line) is a setup in which both the focal actor and his or her schoolmate have medium academic achievement. ${ }^{7}$ Higher contributions to the evaluation function indicate higher probabilities of friendship formation, compared to this medium/medium combination.

In Fig. 3, we see that across the entire spectrum of academic achievement, the evaluation function peaks if ego and alter are maximally similar, i.e., if ego's and alter's average grades are identical. This, again, shows that students are most likely to befriend those peers who are most similar to themselves in their academic achievement. By contrast, friendship formation becomes less likely with increasing differences in ego's and alter's academic achievement. Taken together, these observations reflect the positive achievement similarity effect from Table 1 discussed earlier and show that given the opportunity, all else equal, students most likely select friends who do similarly well in school.

In the influence part of the model, we investigate how actors choose their academic achievement. The rate parameters are estimated at around one, indicating that every actor, on average, has one opportunity to change his or her academic achievement. The results in Table 1 first show that, in the data at hand, boys and girls on average do not differ in their academic achievement, as the respective effect is close to zero and not statistically significant. Furthermore, the linear and quadratic shape effects suggest that there is no substantial tendency for achievement to improve or deteriorate over time. Most importantly, the average achievement similarity effect models how students' academic achievement depends on their social network. Specifically, the effect assesses whether students assimilate their friends' academic achievement over time, i.e., whether there is an influence effect for academic achievement. In our model, the average achievement similarity effect is positive and statistically significant. This indicates that students indeed adapt to their friends' academic achievement, i.e., that they become more similar to them over time.

In other statistical approaches, we would have been unsure about how trustworthy our estimates of the influence effects are, as most of them empirically capture students' similarity in academic achievement when trying to estimate influence. However, as shown above, similarity can also be a consequence of actors' choice to befriend similar-achieving peers. In the SAOM approach, we do not face this problem because selection and influence effects are modelled and estimated jointly from the same model. Therefore, our estimates of influence are net of selection and vice-versa, making SAOMs particularly suitable to study how individual choices are shaped by social networks.

\section{Discussion}

In order to increase the mutual appeal between the fields of social network analysis and choice modelling, we discussed the stochastic actor-oriented model for the co-evolution of networks and behavior (SAOM; Snijders et al., 2010) as a powerful statistical tool to analyze choices in social networks. SAOMs can be used to study two kinds of choice processes as well as their interplay: On the one hand, individuals choose their social relationships (selection); on the other hand, individuals choose to change their behavior in response to the characteristics of their social networks (influence).

In our presentation, we showed that the core of a SAOM is similar to the random utility maximization model (RUM; McFadden, 1973). However, SAOMs provide a way to face the criticism that choice modelling applications often fail to account for the social context in which people make their choices (Bruch and Feinberg, 2017) by allowing to investigate how social networks influence the choices of their members. Furthermore, they allow to understand how interdependent individual tie formation decisions created these social contexts in the first place. We illustrated how SAOMs can be used to study both types of choices with an empirical example of friendship making and academic achievement in the school context, showing that students both choose peers as friends who are similar in academic achievement to them and adjust their academic achievement towards that of their friends.

Our presentation sought to explain and discuss the basic specification of SAOMs. It is important to note, however, that SAOMs can be extended with regard to the types of relationships, the types of actors, and the types of behavior under consideration. Regarding the types of relationships, researchers may use SAOMs to model the simultaneous evolution of multiple networks (so-called multiplex networks) and their effects on each other (e.g., Boda (2018) uses both friendships and race attributions as two different networks). With regard to types of actors, SAOMs are capable of investigating two-mode networks, in which a single network features two types of "actors" and relationships only exist between these types (e.g., in a study by Benton (2016), directors are connected to each other through firms). With regard to the type of behavior investigated, SAOMs may model a range of behavioral variables with different properties. For example, in studies on the diffusion of innovations, the behavioral variable may be binary, with people either adopting an innovation or not adopting it (e.g., Greenan (2015) models the initiation of cannabis smoking as an innovation) or, as proposed recently, it may be measured continuously (Niezink et al., 2019). The frequently updated manual of RSiena (Ripley et al., 2019) guides researchers on how to implement these extensions.

Researchers who consider using SAOMs for studying choices have to be aware that SAOMs impose certain restrictions on the data and are not equally suitable to all kinds of social networks. First, with regard to missing data, social network data should be as complete as possible, which means that both unit and item non-response rates should be rather low (Huisman and Steglich, 2008). Second, as SAOMs were designed to investigate the evolution of social networks, researchers need to have longitudinal network information. While the precondition is to have network observations for at least two time points, SAOMs may also be used to study network

\footnotetext{
7 Notice, however, that in a mini-step the total value of the evaluation function of a network state is calculated based on all effects included in the model specification. In this illustration, however, we are only interested in how friendship formation depends on academic achievement, which is why we only focus on the joint contribution of the achievement-related effects to the value of the evaluation function, holding all other characteristics constant.
} 
evolution if data on a larger number of time points is available. From three time points onwards, researchers can decide whether they want to obtain average model results across all time points or whether they prefer separate model results for transitions between subsequent time points. Third, the assumption of full information in the choice modelling process is likely to be violated in very large networks, which is why SAOMs may be less applicable to the analysis of large-scale social networks such as Twitter, Facebook, patent, or citation networks. In addition, in principle, SAOMs may be extended to feature other forms of choice modelling.

Taken together, we have shown that SAOMs constitute a theory-driven statistical model for studying both selection-the choice of specific network partners - and influence - the effects of social networks on individual choice behavior. Since SAOMs are not bound to specific topics or research questions, and given their similarities with the RUM, they should be useful for the choice modelling community for empirically investigating network-related choices.

\section{Acknowledgements}

This research was supported by grants from the German Research Foundation (DFG/KA 1602/6-1 and 6-2). Part of this work was performed on the computational resource bwUniCluster funded by the Ministry of Science, Research and the Arts Baden-Württemberg and the Universities of the State of Baden- Württemberg, Germany, within the framework program bwHPC.

\section{Appendix A. Preconditions for social network analysis}

An informed statistical investigation of a social network (Wasserman and Faust, 1994) forces researchers to clearly specify both who the members of the network are and the type of relationship that links them to each other.

The first task refers to the so-called boundary specification problem (Laumann et al., 1989). It asks researchers to state who is a member of a social network, and who is not. The boundaries of a network may be institutionally defined by considering all students within a school or every worker within a firm. In the jargon of the social network perspective, the network members may be referred to as "vertices" or "nodes", or, given they have agency, as "actors".

The second task asks researchers to specify the type of relationship that links the network members to each other. Examples for different types of relationships are friendships, romantic relationships, citations, exchanges of goods and services, or measures of the frequency of contact. The actual instantiations of such relationships among the network members may be called "edges", "arcs, or "links", or, if agency is to be highlighted, "ties". Relationships may be directed or undirected; for example, a tie in a scientific collaboration network is undirected because cooperation binds scientists together, but a scientific citation network is directed, with one paper citing another without necessarily being cited back. In a directed relationship, the sender of the tie is called "ego" and the receiver "alter".

Network members and relationships jointly define the social network of interest. Given that the evolution of a social network is subject to the analysis, the network has to be observed empirically at least at two points in time for a SAOM analysis to apply. ${ }^{8}$

\section{References}

Amati, V., Schonenberger, F., Snijders, T.A.B., 2015. Estimation of stochastic actor-oriented models for the evolution of networks by generalized method of moments. J. Soc. Fr. Stat. 156, 140-165.

An, W., 2015. Multilevel meta network analysis with application to studying network dynamics of network interventions. Soc. Netw. 43 , 48-56.

Arentze, T.A., Kowald, M., Axhausen, K.W., 2013. An agent-based random-utility-maximization model to generate social networks with transitivity in geographic space. Soc. Netw. 35, 451-459.

Benton, R.A., 2016. Corporate governance and nested authority: cohesive network structure, actor-driven mechanisms, and the balance of power in American corporations. Am. J. Sociol. 122, 661-713.

Block, P., Grund, T., 2014. Multidimensional homophily in friendship networks. Netw. Sci. 2, 189-212.

Block, P., Heathcote, L.C., Heyes, S.B., 2018. Social interaction and pain: an arctic expedition. Soc. Sci. Med. 196, 47-55.

Boda, Z., 2018. Social influence on observed race. Sociol. Sci. 5, 29-57.

Brandes, U., Indlekofer, N., Mader, M., 2012. Visualization methods for longitudinal social networks and stochastic actor-oriented modelling. Soc. Netw. 34, 291-308. Bruch, E., Feinberg, F., 2017. Decision-making processes in social contexts. Annu. Rev. Sociol. 43, $207-227$.

Calastri, C., Hess, S., Daly, A., Carrasco, J., Choudhury, C.F., 2018. Modelling the loss and retention of contacts in social networks: the role of dyad- level heterogeneity and tie strength. J. Choice Model. 29, 63-77.

Doreian, P., 1989. Network autocorrelation models: problems and prospects. Spat. Stat.: Past, Present, Future 369-389.

Dunbar, R.I., 1992. Neocortex size as a constraint on group size in primates. J. Hum. Evol. 22 (6), 469-493.

Ferligoj, A., Kronegger, L., Mali, F., Snijders, T.A., Doreian, P., 2015. Scientific collaboration dynamics in a national scientific system. Scientometrics 104, 985-1012. Finger, K., Lux, T., 2017. Network formation in the interbank money market: an application of the actor-oriented model. Soc. Netw. 48, $237-249$.

Fortuin, J., Geel, M.V., Vedder, P., 2016. Peers and academic achievement: a longitudinal study on selection and socialization effects of in-class friends. J. Educ. Res. 109, 1-6.

Gasparrini, A., Armstrong, B., Kenward, M.G., 2012. Multivariate meta-analysis for non-linear and other multi-parameter associations. Stat. Med. 31, 3821-3839. Greenan, C.C., 2015. Diffusion of innovations in dynamic networks. J. R. Stat. Soc. Ser. A 178, 147-166.

Hess, S., Daly, A., Batley, R., 2018. Revisiting consistency with random utility maximization: theory and implications for practical work. Theory Decis. 84, 181-204. Huisman, M., Steglich, C., 2008. Treatment of non-response in longitudinal network studies. Soc. Netw. 30, $297-308$.

\footnotetext{
${ }^{8}$ However, although SAOMs were developed to investigate the evolution of network structures, they may also be employed to model crosssectional networks (see Snijders and Steglich, 2015).
} 
Indlekofer, N., 2014. Methods for Diagnosis and Interpretation of Stochastic Actor-Oriented Models for Dynamic Networks. Dissertation, University of Konstanz. Kandel, D.B., 1978. Homophily, selection, and socialization in adolescent friendships. Am. J. Sociol. 84, 427-436.

Kinne, B.J., 2016. Agreeing to arm: Bilateral weapons agreements and the global arms trade. Journal of Peace Research 53 (3), $359-377$.

Kretschmer, D., Leszczensky, L., Pink, S., 2018. Selection and influence processes in academic achievement-more pronounced for girls? Soc. Netw. 52, 251-260.

Kroneberg, C., Kalter, F., 2012. Rational choice theory and empirical research: Methodological and theoretical contributions in Europe. Annual Review of Sociology 38, 73-92.

Lancaster, K.J., 1966. A new approach to consumer theory. Journal of political economy 74 (2), $132-157$.

Laumann, E.O., Marsden, P.V., Prensky, D., 1989. The boundary specification problem in network analysis. In: Freeman, L.C., White, D.R., Romney, K.A. (Eds.), Research Methods in Social Network Analysis. Routledge, New York, pp. 61-87.

Leszczensky, L., Pink, S., 2015. Ethnic segregation of friendship networks in school: testing a rational-choice argument of differences in ethnic homophily between classroom-and grade-level networks. Soc. Netw. 42, 18-26.

Leszczensky, L., Pink, S., 2019. What drives ethnic homophily? A relational approach on how ethnic identification moderates preferences for same-ethnic friends. Am. Sociol. Rev. 84, 394-419.

Leszczensky, L., Pink, S., Kalter, F., 2015. Friendship and Identity in School: Field Report on Wave 1, Wave 2, and Wave 3 (Technical Report), vol. 161. Arbeitspapiere/Mannheimer Zentrum für Europaische Sozialforschung, Working papers.

Lewis, K., Kaufman, J., Gonzalez, M., Wimmer, A., Christakis, N., 2008. Tastes, ties, and time: a new social network dataset using Facebook.com. Soc. Netw. 30, 330-342.

Liebe, U., Mariel, P., Beyer, H., Meyerhoff, J., 2018. Uncovering the nexus between attitudes, preferences, and behavior in sociological applications of stated choice experiments. Sociol. Methods Res. 1-38.

Louviere, J.J., Hensher, D.A., Swait, J.D., 2000. Stated Choice Methods: Analysis and Applications. Cambridge university press.

Maness, M., 2020. Choice modeling perspectives on the use of interpersonal social networks and social interactions in activity and travel behavior. In: Goulias, K.G., Davis, A.W. (Eds.), Mapping the Travel Behavior Genome. Elsevier, pp. 399-411.

McFadden, D., 1973. Conditional logit analysis of qualitative choice behavior. In: Zarembka, P. (Ed.), Frontiers in Econometrics. Academic Press, New York, pp. 105-142.

McFadden, D., 1986. The choice theory approach to market research. Marketing science 5 (4), $275-297$.

McPherson, M., Smith-Lovin, L., Cook, J.M., 2001. Birds of a feather: homophily in social networks. Annu. Rev. Sociol. 27, 415-444.

Mood, C., 2010. Logistic regression: why we cannot do what we think we can do, and what we can do about it. Eur. Sociol. Rev. 26, 67-82.

Niezink, N.M., Snijders, T.A., 2017. Co-evolution of social networks and continuous actor attributes. The Annals of Applied Statistics 11 (4), $1948-1973$.

Niezink, N.M.D., Snijders, T.A.B., van Duijn, M.A.J., 2019. No longer discrete: modeling the dynamics of social networks and continuous behavior. Sociol. Methodol. 49 (1), 295-340 (online first).

Osgood, D.W., Ragan, D.T., Wallace, L., Gest, S.D., Feinberg, M.E., Moody, J., 2013. Peers and the emergence of alcohol use: influence and selection processes in adolescent friendship networks. J. Res. Adolesc. 23, 500-512.

Paez, A., Scott, D.M., 2007. Social influence on travel behavior: a simulation example of the decision to telecommute. Environ. Plan.: Econ. Space 39, 647-665.

Reed, M., 2015. Social network influence on consistent choice. J. Choice Model. 17, 28-38.

Ripley, R.M., Snijders, T.A., Boda, Z., Voros, A., Preciado, P., 2019. Manual for RSiena, Version December 2, 2019. University of Oxford, Department of Statistics, Nuffield College.

Rothenberg, T.J., 1971. Identification in parametric models. Econometrica 39, 577-591.

Snijders, T.A.B., 1996. Stochastic actor-oriented models for network change. J. Math. Sociol. 21, 149-172.

Snijders, T.A.B., 2001. The statistical evaluation of social network dynamics. Sociological methodology 31 (1), 361-395.

Snijders, T.A.B., 2004. Explained variation in dynamic network models. Math. Soc. Sci. 168, 31-41.

Snijders, T.A.B., Steglich, C.E., 2015. Representing micro-macro linkages by actor-based dynamic network models. Sociol. Methods Res. 44, $222-271$.

Snijders, T.A.B., van de Bunt, G.G., Steglich, C.E., 2010. Introduction to stochastic actor-based models for network dynamics. Soc. Netw. 32, 44-60.

Steglich, C., Snijders, T.A.B., Pearson, M., 2010. Dynamic networks and behavior: separating selection from influence. Sociol. Methodol. 40, $329-393$.

Van de Bunt, G.G., van Duijn, M.A.J., Snijders, T.A.B., 1999. Friendship networks through time: an actor-oriented dynamic statistical network model. Comput. Math. Organ. Theor. 5, 167-192.

Veenstra, R., Dijkstra, J.K., Steglich, C., Van Zalk, M.H., 2013. Network-behavior dynamics. J. Res. Adolesc. 23, $399-412$.

Walker, J.L., Ehlers, E., Banerjee, I., Dugundji, E.R., 2011. Correcting for endogeneity in behavioral choice models with social influence variables. Transp. Res. Part A 45, 362-374.

Wasserman, S., Faust, K., 1994. Social Network Analysis: Methods and Applications. Cambridge University Press, Cambridge. 